\title{
STATIC AND DYNAMIC RESPONSES OF SLOWLY ADAPTING JOINT RECEPTORS
}

\author{
W. D. MCCALL, JR., M. C. FARIAS, W. J. WILLIAMS AND S. L. BEMENT
}

Bioelectrical Sciences Laboratory, Electrical and Computer Engineering Department and the Bioengineering Program, University of Michigan, Ann Arbor, Mich. 48105 (U.S.A.)

(Accepted October 24th, 1973)

\section{SUMMARY}

(1) A systems analysis approach was used to study slowly adapting knee joint receptors. Transfer functions were obtained from the receptor response to sinusoidal inputs of knee joint angle over a range of frequencies from 0.01 to $7 \mathrm{~Hz}$.

(2) The Bode plots obtained in the dynamic studies revealed a high pass filter characteristic with a magnitude slope on the order of $5 \mathrm{~dB} /$ decade and a phase lead on the order of $22.5^{\circ}$. The gain of the transfer function was found to be influenced by both the input angle excursion and the static bias angle.

(3) The static angle sensitivity curves of the receptors were found to be "bell shaped' in some cases and to increase or decrease monotonically with joint angle in other cases.

(4) The static sensitivity curve produced by a series of positive increments of joint angle was not the same as the static sensitivity curve produced by a series of negative increments of joint angle. Tentatively this finding is attributed to a nonNewtonian viscoelastic effect and is felt to limit the information available from a single, peripheral receptor.

\section{INTRODUCTION}

In spite of the important role of the joint receptor system as a primary sensory modality, the properties of the several types of receptors involved, their functional role in proprioception, and the many anatomical details of their sensory projections are not well understood. In our laboratory we are investigating the static and dynamic response properties of slowly and rapidly adapting joint receptors at the knee joint level, the level of the dorsal column nuclei, and the level of the thalamic nuclei. This paper reports the results of a quantitative study of the slowly adapting knee joint receptor in the cat at the primary or receptor level.

Muscle spindle $18,21,27,29,31$ and Golgi tendon organ ${ }^{14-16}$ responses have already 
been analyzed extensively and quantitatively. These receptor systems are known to signal position, velocity, and force information from the muscle to spinal and higher levels, especially the cerebellum. The joint receptor afferents project information to many levels of the CNS as well and the joint receptor system is thought to be the primary mediator for the perception of position ${ }^{24}$.

Previous investigations $1,3,4,11,30$ have shown that the static response provides only one part of the response profile and that dynamic analysis is necessary to characterize the receptor response fully. Our purpose was to examine the dynamic properties of the slowly adapting cat knee joint receptors. The results delineate some of the parameters and restrictions imposed on CNS angle and velocity appreciation by the receptor response.

The static properties of the slowly adapting knee joint receptor have been described by several investigators ${ }^{3,4,10,11,30}$. In some reports ${ }^{1,4}$ the static response characteristic (as a function of joint angle) is monotonic and in other reports ${ }^{30}$ it is distinctively 'bell shaped'. A 'bell shaped' response has its maximum response at a particular joint angle; on either side of this angle the response of the receptor decreases. For sufficiently large deviations on either side of the maximum response angle, the receptor response falls to zero.

The transient response of slowly adapting joint receptors has been studied by Boyd and Roberts ${ }^{4}$ and Vierstein ${ }^{32}$. These investigators used saturating ramp inputs of joint angle. Their transient response exhibits a proportional plus derivative type characteristic common to many slowly adapting mechanoreceptors.

Sinusoidal stimuli have been used profitably in the studies of muscle spindle ${ }^{18}$, 21,27 and invertebrate mechanoreceptors ${ }^{2,5,8}$. Our investigation appears to be the first reported effort to use sinusoidal joint motion to extract the dynamic response characteristics of joint receptors. Preliminary results have been presented ${ }^{22}$.

\section{METHODS}

\section{Experimental methods}

Dissection procedure. Eighty cats were used in this study. Single tonic units were recorded successfully in 20 cats. Phasic units were obtained in 52 experiments, to be reported later. Usually only one unit was studied in each cat because of the length of the experimental procedure.

Each animal was anesthetized with sodium pentobarbitol $(35 \mathrm{mg} / \mathrm{kg})$ given intraperitoneally; the cephalic vein was cannulated for further intravenous doses as necessary. The rectal temperature was monitored and maintained at $37.5 \pm 1{ }^{\circ} \mathrm{C}$ with a heating pad.

The lateral side of the femur was exposed and pinned with steel nails. An incision was made on the medial side of the leg parallel to the femur and the sartorius muscle was removed. The femur pins were clamped in the fixture, the skin sewn to a ring, and the resulting pool filled with mineral oil. No attempt was made to maintain the temperature of the mineral oil pool. The cat lay on its side so that the leg was moved in a horizontal plane. 


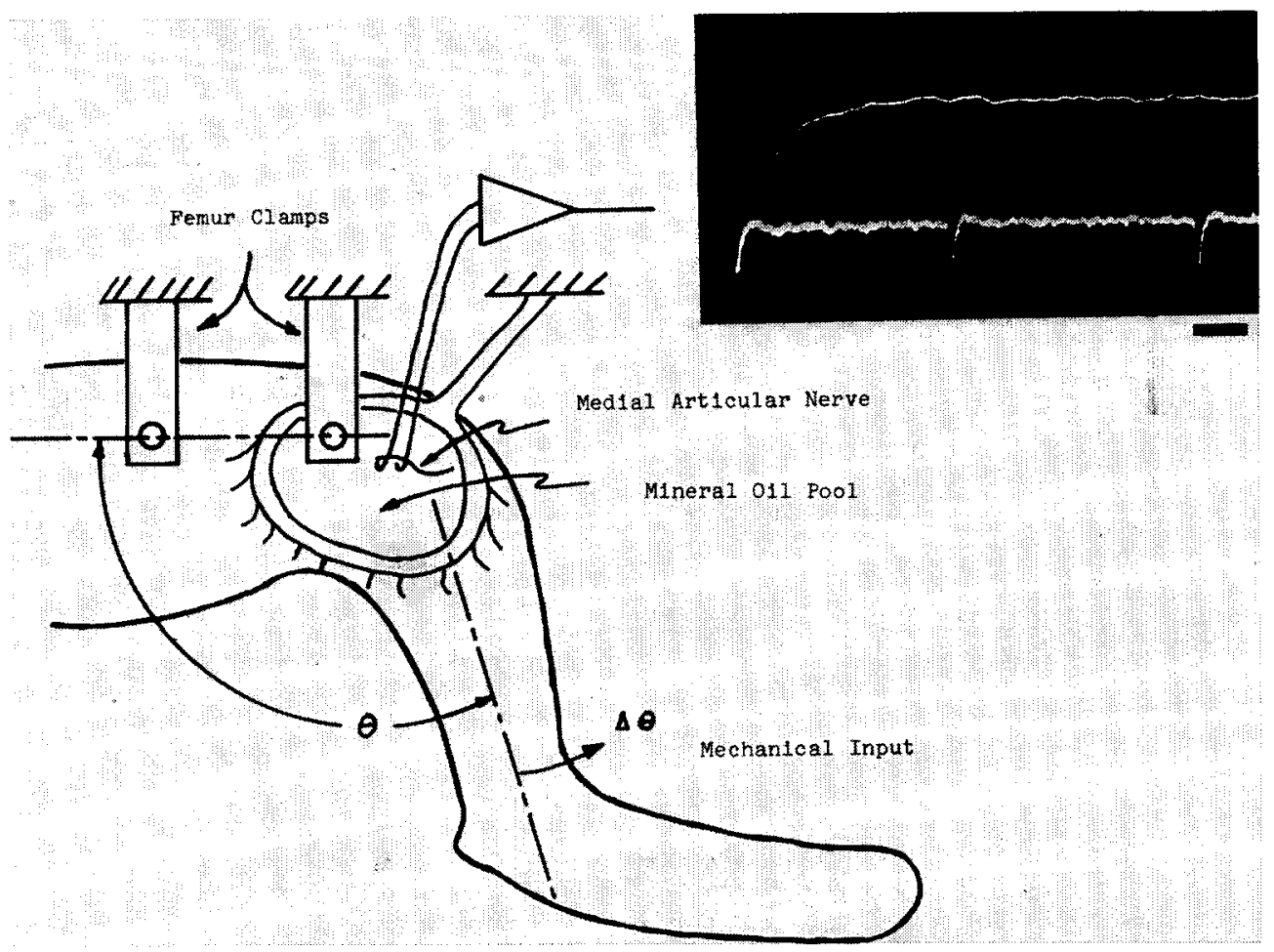

Fig. 1. Schematic drawing of the leg mounting arrangement. The surgical procedures are described in the text. The bias angle, $\theta$, and the amplitude of the sinusoidal excursions, $\Delta \theta$ are defined in the diagram. The electrodes were of silver wire, soldered to the center conductors of two conductor shielded wires with only $2 \mathrm{~cm}$ of the electrodes unshielded. Typical action potentials are shown in the inset. Time calibration: upper trace, $0.5 \mathrm{msec}$; lower, trace $10 \mathrm{msec}$. Occasionally two or more units were recorded. Then a window discriminator was used to select the action potential of interest.

The medial articular nerve runs with the genu suprema artery and vein parallel to the femur. Both blood vessels and the nerve are buried in connective tissue. The nerve was teased away from the connective tissue and the blood vessels, and placed over silver recording electrodes. When necessary the nerve was split with fine forceps to reduce the activity to that of single units. The details of the leg mounting and recording electrodes are shown in Fig. 1.

In a few experiments a laminectomy and extensive denervation were performed and responses recorded from dorsal root filaments. In these instances the cat was mounted in an upright position and the tibia was moved in a vertical plane.

Recording system. A standard system for recording extracellular action potentials was used, as shown in Fig. 2. The recording electrodes were connected to a preamplifier (Tektronix 122). The output of the preamplifier was displayed on an oscilloscope (Tektronix 502A) and the output of the vertical plate amplifier was used as an AM input to an AM/FM tape recorder (Hewlett Packard Model 3960). The bandwidth of the neural recording system was from $80 \mathrm{~Hz}$ to $3.75 \mathrm{kHz}$. The driving voltage to the linear actuator and the output from the knee position potentiometer were 


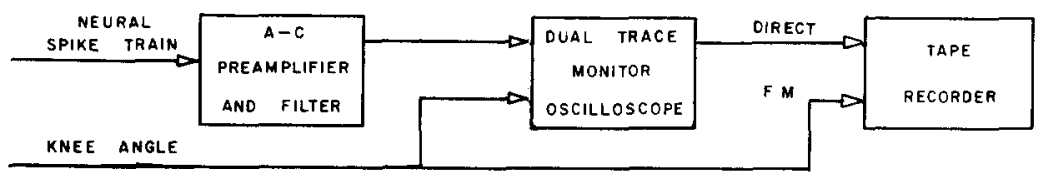

(A) RECORDING

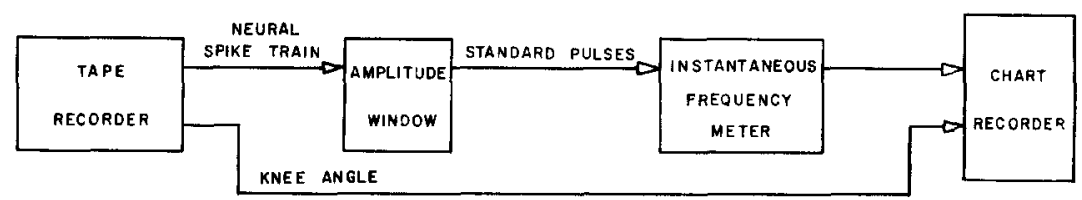

(B) ANALYSIS USING A FREQUENCY METER

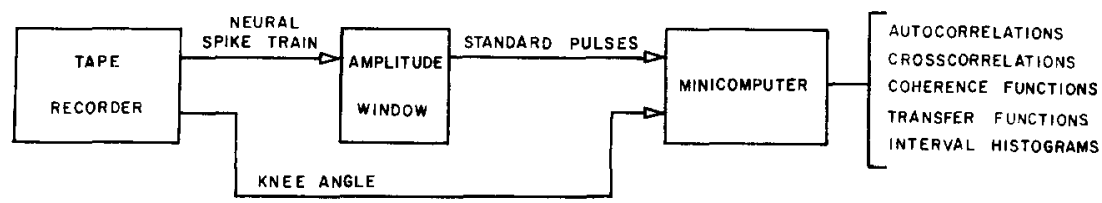

(C) COMPUTER ANALYSIS

Fig. 2. Block diagrams of the recording and analysis arrangements. A : recording. The extracellular neural signal was amplified, filtered (Tektronix 122, $80 \mathrm{~Hz}-10 \mathrm{kHz}$ ), and monitored on an oscilloscope (Tektronix 502A). The vertical signal output of the oscilloscope was recorded on magnetic tape by a Hewlett Packard 3960 Tape Recorder (bandwidth: $50 \mathrm{~Hz}-3.75 \mathrm{kHz}$ ). A voltage proportional to knee angle was also monitored and recorded. B: frequency meter analysis. Single unit responses, isolated by an amplitude window discriminator, were converted to an analog measure of instantaneous frequency by an instantaneous frequency meter. The instantaneous frequency and the knee angle were displayed on adjacent channels of a chart recorder. Gain and phase were measured by hand from the chart record. C: computer analysis. The standard pulses from the amplitude window discriminator were converted from analog to digital form, and the various functions computed, by a digital computer (Hewlett Packard 2115A).

recorded on the FM channels of the tape recorder. Typical recorded action potentials are shown in the inset of Fig. 1.

Mechanical input system. The knee angle variations were provided by coupling a linear actuator ${ }^{13}$ to a knee rotation fixture. The fixture was essentially a hinge with one hasp rigidly clamped and the other hasp attached to the cat ankle. The axis of rotation of the hinge was aligned with the axis of rotation of the knee. The linear actuator was connected to the fixture near the ankle and the knee angle was measured between femur and tibia by a potentiometer mounted on the hinge.

To summarize, the medial articular nerve was exposed, or a laminectomy was performed and dorsal root filaments were isolated. Extracellular action potentials were amplified and recorded on magnetic tape. The knee joint was articulated by a linear actuator and the knee joint angle was also recorded on tape.

\section{Experiments performed}

Two types of experiments were performed, categorized as static and dynamic. In all experiments, the input is considered to be the angle of the tibia with respect to 
the femur, i.e. the particular tibia-femur angle or change of angle. The output is the frequency of the neural spike train from the knee joint receptor.

Static experiments. The protocol was to set the knee at a particular angle, record the adapted neural firing rate, carefully move a small increment (usually 1 or $2^{\circ}$ ) to a new angle, then record the adapted frequency again. When the receptor stopped firing or mechanical impediment to further travel was encountered, the procedure was stopped or repeated in the opposite direction. A 2-min pause between increments was usual, but pauses of $10 \mathrm{~min}$ were also used. The frequency at the end of the pause was taken as the adapted static frequency.

Dynamic experiments. The usual input for dynamic experiments was sinusoidal variation of knee joint angle at a constant excursion but changeable input frequency. The frequency response (Bode plot) for the slowly adapting receptors was obtained in two different, but related ways: with discrete sinusoidal inputs and with swept sinusoidal inputs. In the early experiments we obtained data for several cycles at a particular input frequency. The procedure was repeated for a series of different input frequencies, with the peak-to-peak amplitude of the input angle held constant. The bias angle, or the angle of the knee about which the input sinusoid varied ( $\theta$ in Fig. 1), was also held constant for a particular series of inputs. Triangular inputs were also used to examine certain features of the receptor response.

In later experiments, a sinusoidal input of constant amplitude but changing frequency was used. The input frequency was slowly changed from 0.01 to $3 \mathrm{~Hz}$, or from 0.1 to $7 \mathrm{~Hz}$ in a logarithmic fashion. This technique was fully explained elsewhere ${ }^{34,35}$. The discrete and swept sinusoid techniques gave comparable results when used on the same unit.

The upper and lower frequency limits of sinusoidal input signals were determined by physical limitations. The period of a $0.001 \mathrm{~Hz}$ sinewave is $1000 \mathrm{sec}$ or $16.67 \mathrm{~min}$. If several cycles of data are taken at this frequency a large amount of experimental time is consumed in the process. In addition, the variation in the receptor discharge frequency would be very small and difficult to detect at this low frequency of joint motion. For these reasons the usual lower limit of input signal frequency was $0.01 \mathrm{~Hz}$, while $7 \mathrm{~Hz}$ was the upper limit on the input frequency; above this frequency the preparation began to shake so vigorously that recording of neural data was unreliable. Actual mechanical resonance of the fixture-leg combination occurred at a much higher frequency, in the order of $25 \mathrm{~Hz}$. The mechanical and electrical response characteristics of the actuator system were constant over the range of frequencies used in these experiments.

In summary, both static (unchanging) and dynamic (periodically changing) inputs were used. The dynamic inputs include discrete sinusoids, swept sinusoids, and triangles.

\section{Data processing and analysis}

Analysis of static frequency. In most cases single unit recording was accomplished. In cases where 2 or 3 units were recorded simultaneously, an amplitude window discriminator was used to produce a standard pulse each time the amplitude of an 
action potential was within the desired range. The amplitude window discriminator was still used with single unit recordings in order to produce standard pulses. The standard pulses were then used as inputs to the various processing schemes.

The receptors respond with action potentials of constant shape and amplitude as shown in Fig. 1. In the initial portion of the study the output coding variable was considered to be the instantaneous frequency of the spike train ${ }^{23}$. The interspike interval was determined for each action potential pair. This value was inverted to produce instantaneous frequency and the instantaneous frequency value so obtained was stored in a sample-and-hold circuit until the next instantaneous frequency value was computed. Continuous recording of the sample-and-hold circuit output on one channel of a chart recorder and the joint angle on the other channel of the chart recorder produced the input-output data used for analysis of the receptor responses. The device used to convert the spike train into the continuous estimate of instantaneous frequency is called an instantaneous frequency meter ${ }^{17}$.

A computer based interspike interval histogram was developed later in the study. The average frequency of firing and the standard deviation of the average frequency were computed from the interval histogram data. The average frequency obtained from the instantaneous frequency meter and the interval histogram data were found to correspond in all cases. The static frequency data were obtained with both techniques. The interval histogram technique used 500 sequential intervals to produce each interspike interval histogram. Thus the average frequency was based on 500 data values.

Analysis of dynamic frequency. The analysis of the neural responses to dynamic inputs also involved two techniques: the instantaneous frequency meter was used for the discrete sinusoidal and triangular inputs, and a computer technique ${ }^{34,35}$ was used for the swept sinusoidal inputs. In each case the magnitude of the frequency excursions of the output and its phase shift relative to the input were obtained. The coherence function, a measure of the degree to which the output reflects the input signal rather than uncorrelated noise ${ }^{28}$, was also obtained for the computer-based technique.

The Bode plot results were computed 'by hand' from chart recordings of joint angle and instantaneous frequency. The peak-to-peak excursion of the instantaneous frequency output and the peak-to-peak excursion of the joint angle were determined. Twenty times the logarithm to the base 10 of the ratio of the frequency excursion to the joint angle excursion was determined for each discrete input frequency value. These results form the basis for the magnitude portion of the Bode plot. The magnitude ratio (in decibels by virtue of the logarithmic conversion) was plotted against the input frequency. The input frequency was plotted on a logarithmic scale. The phase information was determined by measuring the phase shift in degrees between the joint angle sinusoid and the instantaneous frequency sinusoid. The same two sinusoids used to determine the magnitude portion of the Bode plot were used in the phase determination. The phase in degrees was plotted against frequency where frequency was plotted on a logarithmic scale. Together the magnitude and phase data form the Bode plot.

The same type of results were produced with the swept frequency technique ${ }^{34,35}$. 
In this case the standard pulse train produced by the amplitude window discriminator was 'demodulated' by a low pass filter. The cross power spectral density between the output of the low pass filter and the joint angle input were determined. Also the power spectral density of the joint angle input was determined. Finally, the system transfer function, the ratio of the output-input cross power spectral density to the input power spectral density was determined. The effects of the low pass filter were removed by computation. The resulting transfer function carries the same type of information as one might obtain by the hand calculations described in the previous paragraph. The swept frequency technique produces information equivalent to that obtained from 128 discrete frequency values in our particular case. The discrete frequency method and the swept frequency method will produce the same result with reference to the fundamental component of the output sinusoid. The harmonic distortion of the output sinusoid was very small in most of our results. The swept frequency technique produced results that were equivalent to the hand calculations in this case. (A more complete description of the swept frequency technique has been presented ${ }^{34}$, 35.)

Error estimates. The static input angle was read from a protractor mounted on the cat leg fixture and had a maximum error of about $\pm 0.25^{\circ}$ for each reading. For the sinusoidal input, the peak input amplitude was constant and thus did not influence the Bode plot. For the static data, the successive leg positions were usually set $2^{\circ}$ apart. Thus the error between any two adjacent readings could have been as much as $\pm 25 \%$. However, this maximum error of $0.5^{\circ}$ pertained to any two readings and there was no cumulative error in the static angle measurement.

The phase angle in the discrete sinusoid experiments was computed from the time difference between the peak or zero crossing of the input and output waveforms. The maximum error was estimated to be $\pm 10 \%$ in the determination of the input peak value and $\pm 5 \%$ for the zero crossing value. Estimates for the output phase error were $\pm 5 \%$ for the peak reading and $\pm 3 \%$ for the zero crossing. Thus the maximum error for the phase determination was estimated to be $\pm 15 \%$.

The determination of the amplitude of the output frequency had two error components; non-linearity in the frequency meter and inaccuracy in the chart reading. Both these errors were largest for small amplitude outputs and decreased as the amplitude of the output increased. The chart reading error could have been as much as $5 \%$. The frequency meter was calibrated each day to within $10 \%$ and the deviation was always on one side of the 'true value' line. As a result, the maximum error in any peak-to-peak amplitude measurement was $15 \%$ or less.

The error associated with the swept frequency dynamic results is quite small, with coherence function values usually 0.8 or larger ${ }^{34,35}$. For this value of the coherence function and for the data processing time used our experiments, the $95 \%$ confidence interval of the amplitude and phase uncertainty is approximately twice the width of the symbols used to plot the data, based on simulations and empirical observations. This high degree of accuracy applies only to a given experimental run, of course, and does not guarantee such a high degree of accuracy from experiment to experiment. 
Frequency meter considerations. The frequency meter incorporated a sample and hold circuit that provides a linear output only in the special case of a constant sampling interval $^{19}$. Since the sampling interval in our frequency meter depends on the signal amplitude (i.e. interspike interval), the response is non-linear, and this effect on the experimental data must be carefully evaluated ${ }^{19,20}$. In addition, the amplifiers and pulse-forming circuitry contribute dynamic filtering characteristics. However, a Bode plot of the frequency meter response characteristics indicated that its response was essentially linear for the frequency and amplitude ranges used in our experiments. The dynamic filtering effects of the frequency meter were smaller than the noise variations at the frequencies employed in this study. Therefore modification of experimental data by the frequency meter was considered negligible. Non-linearities also occur when the modulating frequency approaches the carrier frequency of the spike train. However, the modulation frequency was much less than the carrier frequency in all our experiments.

The data obtained with the swept frequency technique are based on the assumption that the time average frequency is the output coding variable. This technique is linear in nature and avoids non-linear effects present in the frequency meter. We do not know if instantaneous frequency, average frequency, or some other coding variable is the relevant variable as far as the nervous system is concerned. However, the instantaneous frequency and average frequency results are quite similar in our case, probably because the input frequency is much less than the average (carrier) frequency of action potentials in these experiments. If the input frequency approaches the carrier frequency, the results obtained with the two methods should diverge sharply and the results should be interpreted with caution.

In summary, both static and dynamic results were analyzed initially 'by hand' from the output of a frequency meter, whereas later analysis was by computer processing. The output variable was taken to be instantaneous frequency when the frequency meter was used and the average frequency when the swept frequency computer processing technique was used. Error estimates were established.

\section{RESULTS}

\section{Static results}

In several cases 'bell shaped' angle sensitivity curves were obtained while in other cases the receptor response increased monotonically until the mechanical limit was reached. The two types of angle sensitivity curves are exemplified in Fig. 3. The monotonic type appeared about twice as often as the bell shaped type. In general more receptors are active near the limits of flexion and extension whereas relatively few receptors are active in the mid-range.

One of the difficulties encountered in the study of the static response characteristics of slowly adapting knee joint receptors involved the time course of receptor adaptation. Even after long periods of adaptation it was at first not certain that the receptor had reached a steady rate of discharge. As one attempted to trace over the same static response curve several times by moving the joint through a series of 


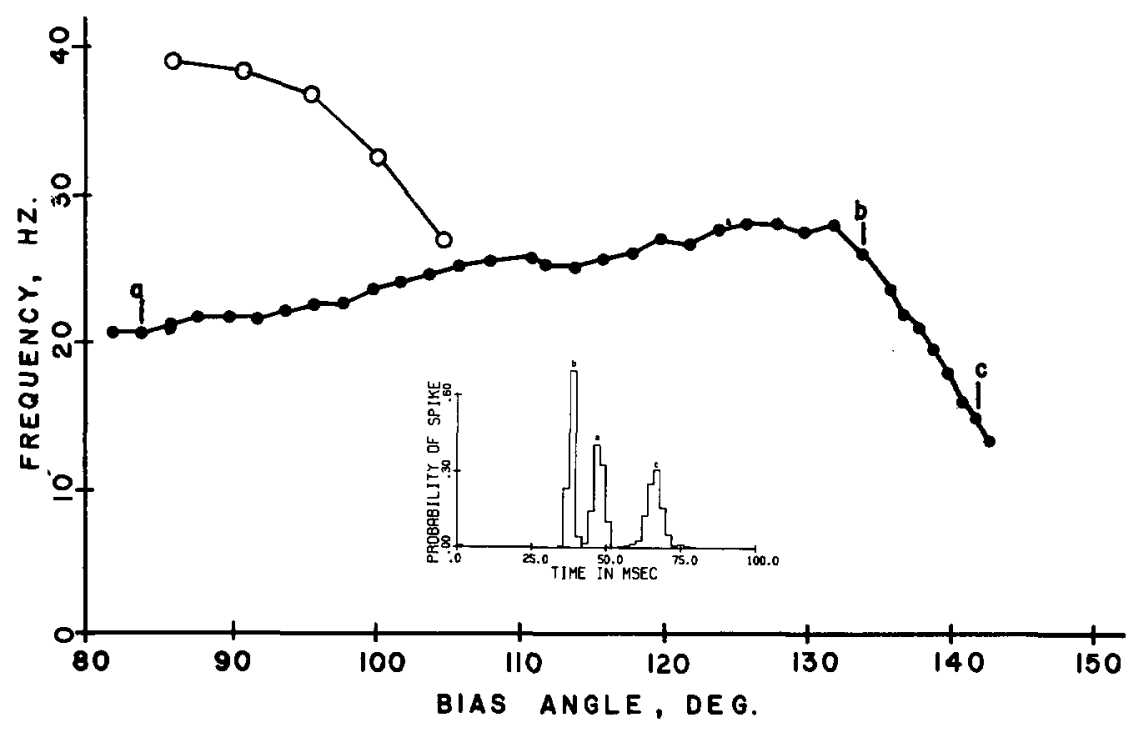

Fig. 3. Static response of two slowly adapting units. Adapted neural frequency plotted against knee angle, where $180^{\circ}$ between femur and tibia would be complete extension. Filled circles: this unit was silent at $144^{\circ}$ and responded as shown when the angle was changed in $2^{\circ}$ increments until $82^{\circ}$, where mechanical interference occurred. The bias angles for the interspike interval histograms in the inset are indicated $a, b$, and $c$. Open circles: the static frequency and frequency response for this unit were both obtained before moving to another bias angle. These static points were obtained after each frequency response at the particular bias angle. Inset: interspike interval histograms. Three histograms, a, b, and c were obtained at bias angles $84^{\circ}, 131^{\circ}$, and $142^{\circ}$ respectively from the response plotted with filled circles. Each histogram was computed from 500 intervals with a bin width of $2 \mathrm{msec}$.

positive increments of angle and then reversing through a series of negative increments of angle, etc., a different static response curve was found when progressing in the positive and negative directions. Initially it was not clear whether this was from a hysteresis type of phenomenon or from a latent adaptive component in the receptor response. The latter possibility was examined by allowing long pauses between increments. Even after $10 \mathrm{~min}$ of adaptation between increments of joint angle it was not possible to retrace the same static curve for both positive and negative increments of joint angle. Fig. 4 shows some typical results obtained with 10 -min pauses.

In order to resolve the question of hysteresis $v s$. long adaptation time, the time course of adaptation to step inputs was examined carefully over a time course of $10 \mathrm{~min}$. Fig. 5 shows a $\log -\log$ plot of output frequency $v s$. time, averaged for 10 sequential spikes at each time shown. Adaptation is evidently complete within the first 2 min. Also shown in Fig. 5 is a dotted line which is the step response predicted by a quarter-order-differentiator model (see Appendix). The quarter-order-differentiator model predicts a straight line with slope $-\mathrm{k}$ when the data are plotted on logarithmic axes.

The inability to retrace the same static response curve must be ascribed to a hysteresis-like effect. The severity of the hysteresis was estimated by measuring the 


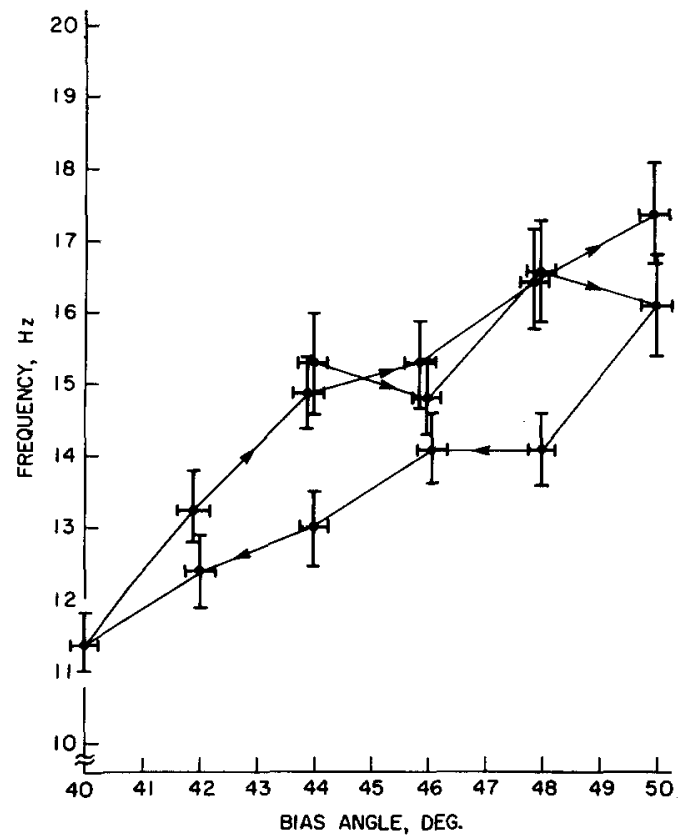

Fig. 4. Static sensitivity curves for increasing and decreasing increments of angle. Starting at a bias angle of $44^{\circ}$, the joint angle was increased in $2^{\circ}$ increments up to $50^{\circ}$. Then the joint angle was decreased by $2^{\circ}$ increments to $40^{\circ}$. Finally the joint angle was again increased by $2^{\circ}$ increments until $50^{\circ}$ was reached. The receptor was allowed to adapt for $10 \mathrm{~min}$ between each angular increment. The vertical error bars indicate one standard deviation on either side of the mean frequency as determined from the interval histogram obtained at each bias angle. The horizontal error bars indicate the estimated maximum angle measurement error.

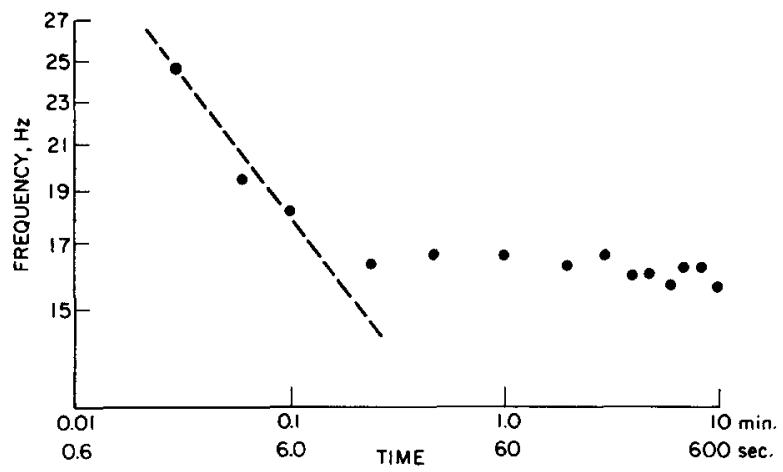

Fig. 5. Frequency vs. time after step. The circles represent neural frequency after a $2^{\circ}$ step change of knee angle. Both horizontal and vertical axes are logarithmic. Each circle is the average of 10 sequential interpulse intervals. The dashed line is the step response predicted by the quarter-orderdifferentiator model discussed in the appendix. These data were obtained from cat 49 . The joint was moved from 46 to $48^{\circ}$. Adaptation is nearly complete after $10 \mathrm{sec}$. 
largest angular ambiguity that could be observed for a given frequency. This severity ranged from $4^{\circ}$ to $10^{\circ}$ and averaged $6.4^{\circ}$. At first thought one may be tempted to ascribe this result to the mechanical fixture and recording technique. The cat limb was attached firmly to the mechanical fixture. The maximum angle off-set between the ascending and descending angle sensitivity curves due to measurement errors is in the order of $0.5^{\circ}$ (see Methods), while the experimental angle off-set between the ascending and descending curves was as much as $6^{\circ}$, much more than can be attributed to the mechanical fixture and recording. Thus the error contributed by the fixture and the technique cannot account for the observed result. The non-repeatability is most likely a manifestation of a non-Newtonian component of viscoelasticity in the joint tissue. Ellis ${ }^{12}$ has observed a similar phenomenon in the mechanical response of cat tendon.
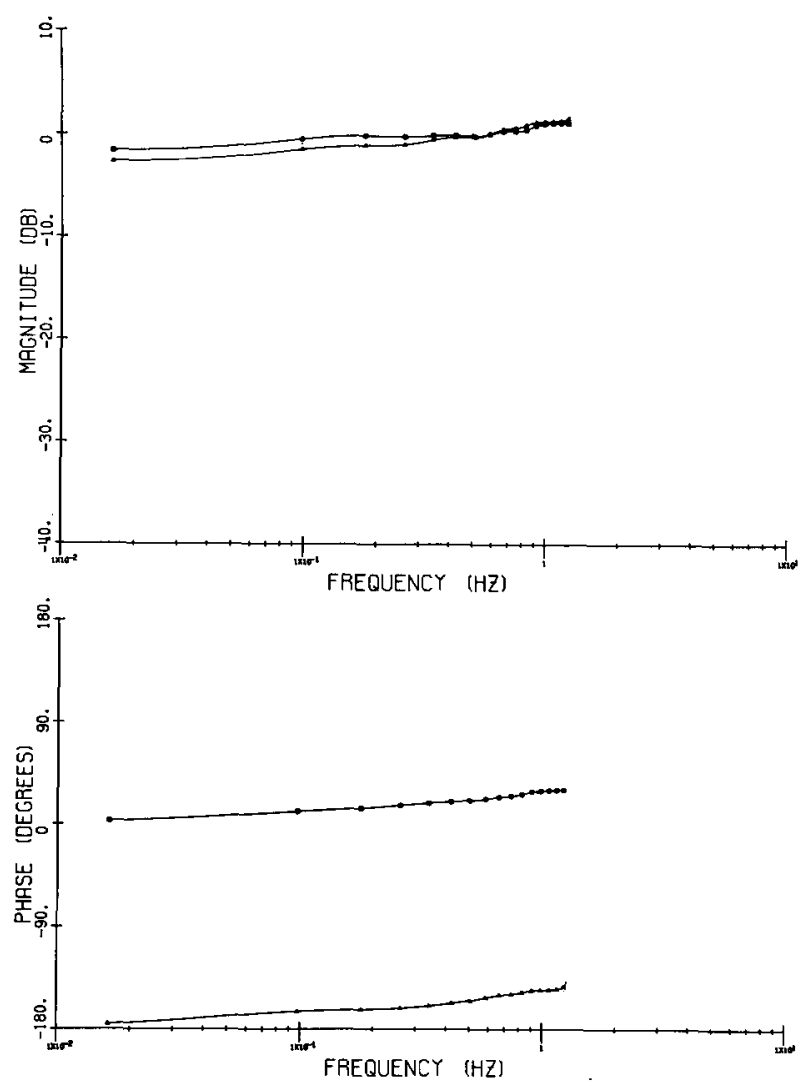

Fig. 6. Effect of bias angle on frequency response. The plot represented by the circles on a solid line was obtained at a bias angle of $103^{\circ}$ (ascending portion of the static sensitivity curve shown as filled circles in Fig. 3). The plot represented by the triangular symbols was obtained at a bias angle of $129^{\circ}$ (descending portion of the static sensitivity curve). Input amplitude was $6^{\circ}$ in both cases. The reversal in slope of the static sensitivity curve accounts for the $180^{\circ}$ difference in the phase angle portions of the two Bode plots. Note the similarity in shape of the two magnitude portions and of the two phase portions of the Bode plots. Zero $\mathrm{dB}$ is arbitrary but consistent for the two curves. 

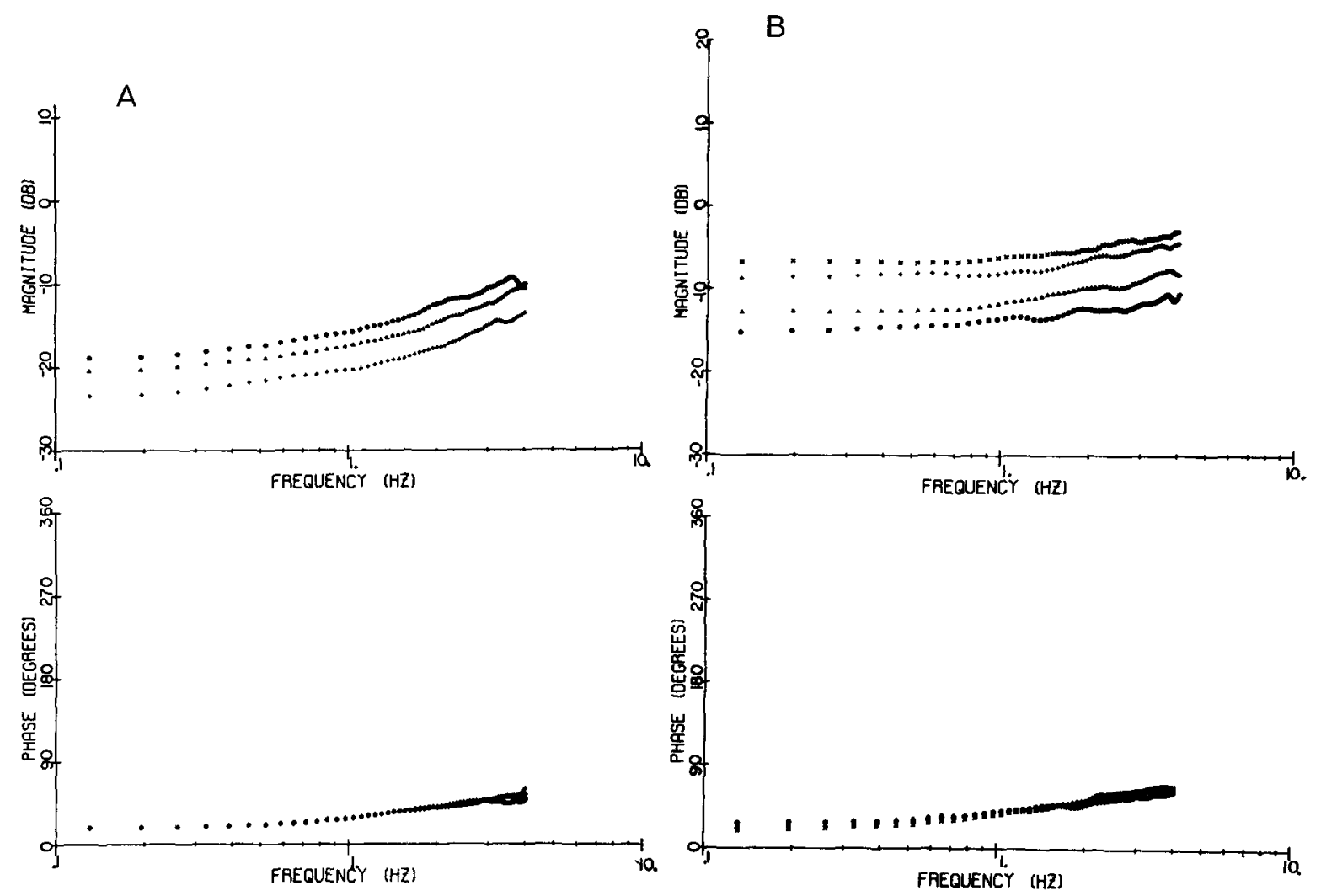

Fig. 7. A: influence of $\theta$ on the frequency response. Three frequency response profiles are shown, corresponding to $\theta=95^{\circ}(\bullet), \theta=91^{\circ}(\Lambda)$ and $\theta=86^{\circ}(+)$, where $\Delta \theta$ was fixed at $4.5^{\circ}$ in each case. Note that the magnitude portion of the plot shifts with different bias angles, but changes in shape minimally. Note also that the phase portions of the 3 Bode plots are nearly identical. The swept frequency technique was used. Zero $\mathrm{dB}$ corresponds to $39.3 \mathrm{imp} . / \mathrm{sec} /$ degree for both $\mathrm{A}$ and $\mathrm{B}$. B: influence of $\Delta \theta$ on the frequency response. In this case $\theta$ was held constant at $104^{\circ}$ and $\Delta \theta$ was changed: $\Delta \theta=2.2^{\circ}(\times), 2.8^{\circ}(+), 3.9^{\circ}(\Lambda)$, and $5.5^{\circ}(\bullet)$. If the system were linear, all Bode plots would superimpose. In this case the dynamic sensitivity (gain) of the receptor decreases with increasing $\Delta \theta$. Note again that the phase portions of the Bode plots are nearly identical.

\section{Dynamic results}

General features. Typical frequency response functions (Bode plots) for the slowly adapting receptors are given in Figs. 6 and 7. These results illustrate several features of the response characteristics.

One basic feature of all of the results was that the magnitude portion of the Bode plot increased monotonically with frequency. The magnitude plot was close to a straight line with a slope of approximately $5 \mathrm{~dB} /$ decade. Refer to the Appendix for a derivation of the quarter-order-differentiator model. A second basic feature was that the phase portion exhibited moderate lead, a fraction of $90^{\circ}$, and increased somewhat with frequency. A third feature was that the slope of the magnitude portion and phase lead at given frequencies were rather invariant under a large range of conditions. Results obtained at the dorsal root level are shown in Fig. 8. As might be 

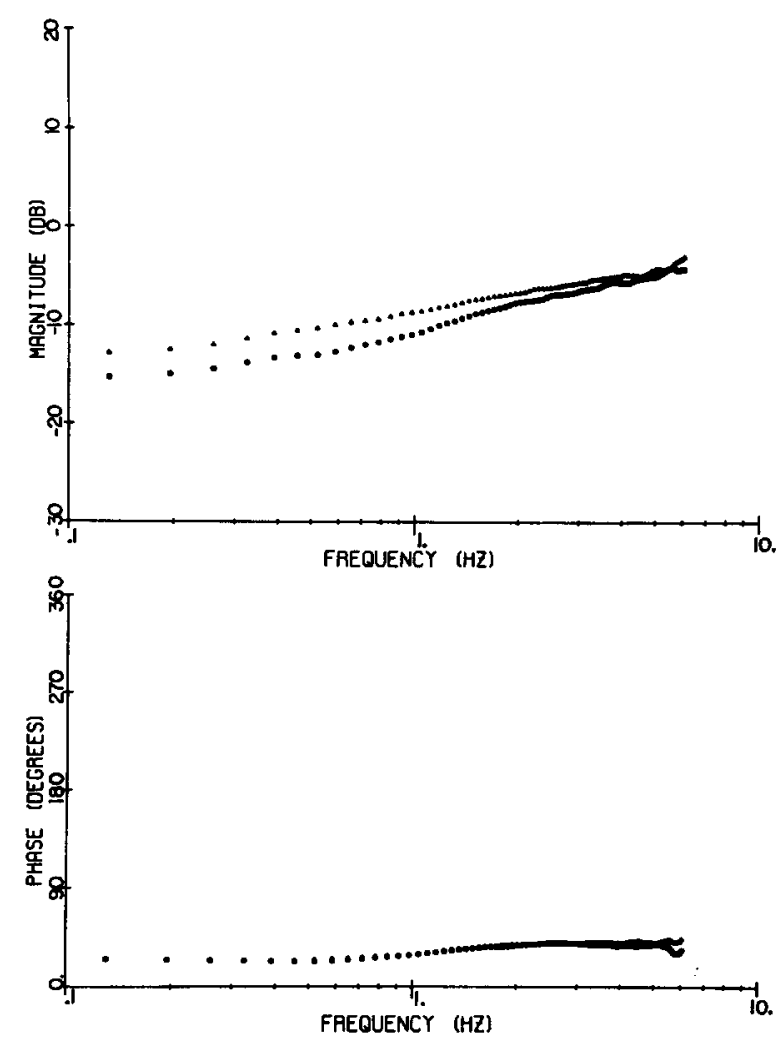

Fig. 8. Frequency response of a slowly adapting unit recorded from dorsal root L7. Standard laminectomy and dissection procedures were used and the leg denervated extensively. The results for two static bias angles are shown. The excursion amplitude was $5^{\circ}$ in both cases. The upper curve $(\Delta)$ was obtained at a bias angle of $95^{\circ}$ and the lower curve (O) was obtained at a bias angle of $85^{\circ}$. Zero $\mathrm{dB}$ corresponds to $30.7 \mathrm{imp} . / \mathrm{sec} /$ degree.

expected, these results are similar to the results obtained at the knee joint level.

The results obtained with a series of discrete sinusoids were similar to the swept frequency results. Averaged results for 6 discrete sinusoid experiments are shown in Fig. 9. Although the spread of these data is greater than for the swept frequency results, the basic finding is very similar: a moderate increase in magnitude with frequency (on the order of $5 \mathrm{~dB} /$ decade) and a phase lead that is a fraction of $90^{\circ}$ and increases with frequency.

Relation to static curve. While the slope and phase of the Bode plot were invariant, the gain of the frequency response function appeared to correspond roughly to the slope of the static response curve at the bias angle where the dynamic experiment was performed. For example, as one proceeds to larger bias angles in Fig. 3 (open circles) the absolute value of the slope increases; similarly, as one proceeds to larger bias angles in Fig. 7A the gain (or vertical level of the magnitude plot) increases. Thus both the static slope and dynamic gain increase with, in this case, increasing bias angle. This was not an unexpected result. Unfortunately the difficulty 

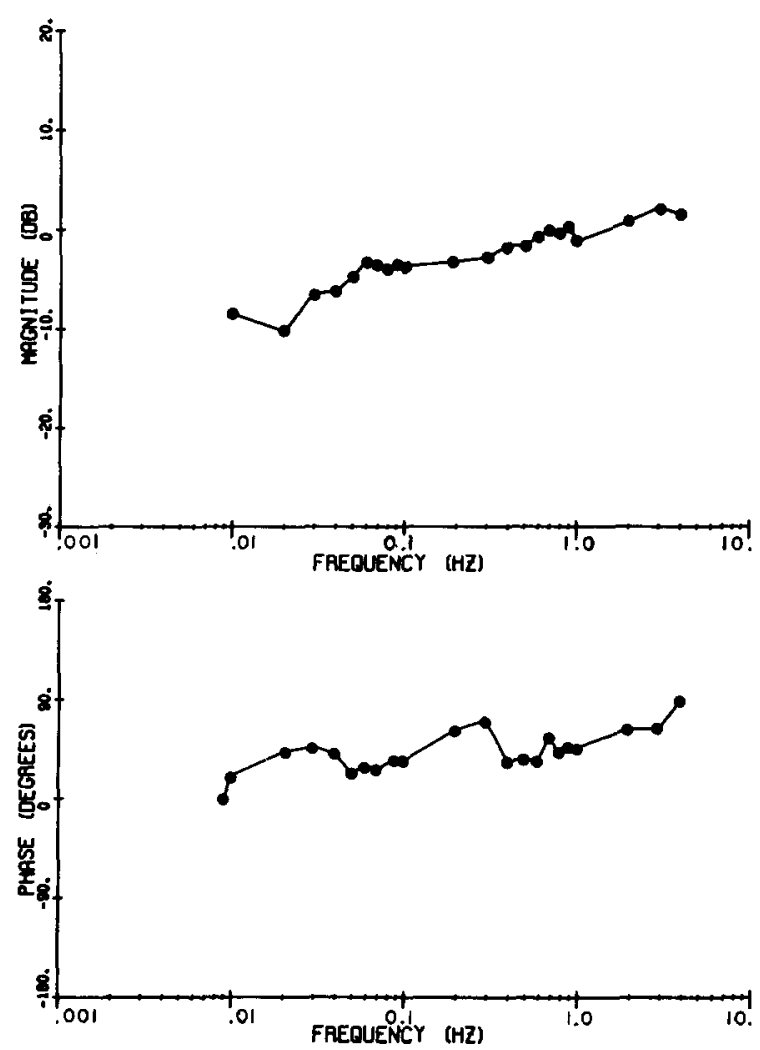

Fig. 9. Frequency response from discrete sinusoids. Data from 6 cats totalling 158 discrete frequencies were averaged. The magnitude part of the frequency response shows an increase with increasing modulation frequency while the phase shows a moderate lead that increases somewhat with modulation frequency. The deviation in these data are large in comparison to the higher reliability of the computer processed results obtained later in the study. Zero dB is arbitrary.

in obtaining the 'true' static response curve of the receptor (because of the suspected non-Newtonian viscoelastic effect) prevented us from obtaining reliable estimates of the slope of the static response curve.

Table $I$ shows some typical system gains for similar carrier frequencies and $\Delta \theta$ values. The gain is defined as the peak-to-peak frequency excursion $\left(f_{p-p}\right)$ divided by the peak-to-peak angle excursion $(\Delta \theta)$.

TABLE I

TYPICAL GAINS FOR A $0.1 \mathrm{~Hz}$ INPUT SINUSOID

\begin{tabular}{lllll}
\hline Cat & $f_{\text {ave }}$ & $f_{p-p}$ & $\Delta \theta$ & $\begin{array}{l}\text { Gain } \\
\text { (pulses/sec/degree) }\end{array}$ \\
\hline 35 & 21 & 13 & $5^{\circ}$ & 2.6 \\
39 & 24 & 18 & $5^{\circ}$ & 3.2 \\
24 & 14 & 18 & $5^{\circ}$ & 3.2 \\
34 & 13 & 20 & $5.5^{\circ}$ & 3.6 \\
\hline
\end{tabular}


The dynamic responses obtained on the ascending and descending portions of the bell shaped curve are compared in Fig. 6 . The shape of the magnitude curves in both cases is similar. The shape of the phase curves is similar, but there is a $180^{\circ}$ phase difference. This $180^{\circ}$ phase shift would be expected if the gain of the response function were related to the slope of the static response curve. The reversal in sign of the slope of the static response curve is reflected as the $180^{\circ}$ shift in the frequency response of Fig. 6.

Non-linearities. In addition to bias dependence, the gain was also found to depend upon the amplitude of the input excursion. Fig. 7B shows this effect. The gain of the frequency response function decreased as the input amplitude increased. This would be expected if a saturation-like non-linearity were present.

An additional type of non-linear behavior was observed in some cases. Neural spikes began to 'drop out' from the receptor response during the negative-going portion of the input. This effect became evident at frequencies as low as $1 \mathrm{~Hz}$ on occasion, and became more pronounced as the input frequency was increased. Sometimes, at the highest input frequency, the receptor output was 'phased-locked' to the input sinusoid such that the receptor fired only a few times per input cycle, at a particular point or phase of the sinusoidal input waveform.

Under such a phase locking condition, the instantaneous frequency meter response is quite non-linear. For this reason we do not process data in this manner beyond the input frequency where phase locking or significant drop-out becomes apparent. All the results presented in this paper were obtained from data that exhibited only minimal 'spike dropping' at higher frequencies and no apparent phase-locking. Furthermore, the harmonic content of the fundamental power present in the neural response was also small, as computed from the coherence function. The fundamental component was usually dominant to the point that harmonic contamination was not evident to visual inspection.

Another non-linearity common to many biological systems is unidirectional rate sensitivity 9 . The response of a unidirectional rate sensitive system is dependent on the sign of the time derivative of the input signal. Such systems exhibit different response characteristics for positive and negative derivatives of the input. If the frequency response function is obtained from a unidirectional rate sensitive system by blind application of sinusoidal system identification techniques, the result is an incomprehensible mixture of the positive and negative rate responses. Thus the presence of appreciable unidirectional rate sensitivity indicates that caution is necessary in the interpretation of results obtained with sinusoidal input signals as tools for system identification ${ }^{9}$.

The triangle wave input can be used as a check for possible unidirectional rate sensitivity, since the respective positive and negative slopes produce rates with equal magnitude but opposite sign. The response instantaneous frequency $v s$. time of the receptors to triangle wave input was examined for a range of positive and negative slopes from 0.5 to $8 \% \mathrm{sec}$. The response was similar for the positive and negative rate portion of the input waveform. Although slight asymmetry was observed in some cases, unidirectional rate sensitivity was not present to a degree sufficient to com- 
promise the generality of the results obtained with sinusoidal input signals.

In summary, the dynamic response from the knee joint receptors was found to contain (1) a consistent frequency response profile with a slowly rising magnitude portion and moderate phase lead, (2) an amplitude non-linearity such that increased input amplitudes resulted in decreased gain, (3) some phase locking in that the response to higher input frequencies tended to occur at only one part of the sinusoidal cycle, and (4) minimal unidirectional rate sensitivity such that responses to triangle waves did not differ markedly for positive and negative slopes.

DISCUSSION

\section{Static results}

In some cases the static angle sensitivity curves of the slowly adapting receptors were found to be similar to the 'bell shaped' curves described by Skoglund ${ }^{30}$. In other cases the static sensitivity curves were found to monotonically decrease or increase as a function of static joint angle, as reported by Andrew and Dodt ${ }^{1}$.

Possibly the bell shaped curves result from twisting the joint as it is articulated. If the bones on both sides of the joint were pinned securely and the joint articulated without twisting, the bell-shaped angle sensitivity curves might not be observed? Perhaps then the angle sensitivity curves would all be similar to Fig. 3 (open circles). The bell shaped curves cannot be considered as artifacts, however, since in normal activity the unrestrained joint probably twists as it is articulated. Thus any rigid mounting scheme that constrained the joint motion to be in a particular plane of rotation might constrain the joint in an artificial manner. Differences in joint loading during flexion and extension of the limb may also be a factor in causing the hysteresis observed in the static data.

A more comprehensive study of the static responses of the receptor under various types of joint articulation and loading is needed to clarify the situation. Ideally an orthogonal set of 3 rotational axes would be defined. The receptor response as a function of all 3 types of rotation and various combinations of these rotations would define completely the static response characteristics of the receptor for a given joint loading. Different joint loadings (e.g. forces chosen to mimic the weight of the cat) would possibly produce different sets of response curves. Clearly this is a problem of some complexity. Recognizing these limitations in our understanding and acquisition of a complete repertoire of static responses, we will discuss our present research findings.

The occurrence of bell shaped curves raises some question as to the way in which the nervous system interprets the information from these tonic receptors. The basic problem is that any bell shaped curve is double-valued so that the receptor responds with the same firing rate at two different joint angles. Thus position information available from only a single receptor would be ambiguous.

Unambiguous position information may still be extracted from the population response of such receptors. A labeled line scheme is one possible method. The static sensitivity curves of cells in the thalamus (thought to respond to slowly adapting joint 
receptors) are monotonic with a maximal response at flexion in some cases and at extension in other cases ${ }^{25,26}$. This seems to support the idea that higher level cells may combine the outputs of a receptor population in such a way as to remove ambiguities present in lower level responses. There may be cells at higher levels that respond selectively to one direction of joint articulation and another group of cells that respond to an opposite direction of joint articulation. This type of processing has been observed at higher levels in the visual system. An investigation directed toward confirmation of such directional sensitivity would require a sophisticated, precise, multi-dimensional joint articulator. Since our present research effort has been directed primarily toward extraction of the dynamic responses of the slowly adapting joint receptors, we must hold open the question of the relationship between the shape of the static sensitivity curves and the handling of the static information by the nervous system.

As previously mentioned, a bell shaped static response is often observed at the primary receptor level and apparently is absent at the tertiary or thalamic level. The processing of slowly adapting information at the secondary neuron then becomes a question of considerable interest. We have searched without success for slowly adapting secondary units in the gracile nucleus, classically held to be the second order relay station $^{33,34}$. Burgess and Clark also reported that only rapidly adapting responses ascended to cervical levels ${ }^{6}$. We await positive anatomical identification of the pathway and location of the second-order cells that respond to the slowly adapting receptor inputs.

The hysteresis-like effect is another puzzling aspect of the slowly adapting recepttor response. This effect is seen in Fig. 16 of Skoglund's primary level data ${ }^{30}$ when these data are plotted on the same axes, and it is also apparent in the thalamic data of Mountcastle ${ }^{25}$. We found that the hysteresis effect persisted even with $10 \mathrm{~min}$ intervals between new static angle settings. Kirkwood's ${ }^{18}$ data from frog muscle spindle experiments exhibit similar hysteresis-like effects. His results indicate that hysteresis is still observed under controlled loading conditions. A long-term adaptation time course such as that suggested by a step response of the form $t^{-0.25}$ (Appendix) could also produce the hysteresis-like effects observed in the data.

Our results (Fig. 4) indicate that the transient response is well modelled by $\mathrm{t}^{-0.25}$ for only the first few seconds. This is similar to the findings of Brown and Stein ${ }^{5}$ for the crayfish stretch receptor. This model is therefore limited in that the transient response is not correctly predicted for long-time periods.

Dynamic results. The consistency of our dynamic results over a large range of static bias angles suggests that these results are not seriously compromised by our incomplete knowledge of the full repertoire of static angle sensitivity curves and their relation to dynamic responses. The fractional order differentiator model used to describe the dynamic response of the slowly adapting receptor is an inexact model, and therefore can represent the real entity only over a necessarily limited range of conditions. We have not yet been able to determine the lower frequency limit of applicability of this model in the joint receptor case. In the frequency range of our investigations, the fractional order differentiator model deviates from the magnitude and phase data in that both the slope of the magnitude portion and the phase lead 
increase moderately with frequency. Also, the fractional order differentiator model suggests that the receptor output should eventually adapt to zero, which we have not observed. Such an adaptation to zero frequency does not seem to be a desirable feature of a candidate position receptor. Can one 'forget' the position of his limb if the limb remains in a static position for a sufficient length of time?

The hysteresis-like effect would appear to compromise monitoring of joint position. A 'dead zone' or zone of ambiguity would exist such that a specific firing frequency could not be precisely related to absolute joint position.

\section{Dynamic models}

The dynamic results consistently suggest a receptor response with a moderate high-pass filtering effect. A fractional order differentiator model fits these data fairly well. Models for other mechanoreceptor types such as Golgi tendon organs ${ }^{14}$ and muscle spindles ${ }^{18,27}$ give similar transfer function predictions.

A comparison of the models of mechanoreceptors suggested by several investigators has been made from the Bode plots predicted by each transfer function (Fig. 10). The appropriate transfer functions are listed in Table II. The Bode plots of all these models bear some similarity to our experimental data, in that they show rising magnitude and increasing phase with frequency. The Houk and Henneman model ${ }^{14}$ for the response of the Golgi tendon organ and the Brown and Stein quarter-orderdifferentiator model $^{5}$ for the response of the crustacean stretch receptors seem to approximate our experimental data more closely than the other two models. The model for slowly adapting joint receptor in monkey suggested by Viernstein ${ }^{32}$ has a
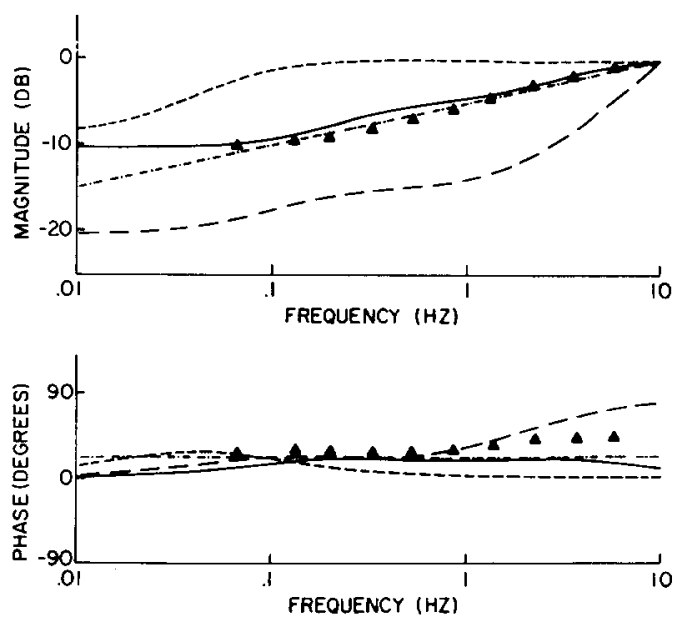

Fig. 10. Comparison of Bode plots for 4 receptor models. Bode plots were generated from 4 mechanoreceptor models: joint receptor (short dashes) as modelled by Viernstein ${ }^{32}$, Golgi tendon organ (solid line) as modelled by Houk and Henneman ${ }^{14}$, secondary muscle spindle (long dashes) by Poppele and Bowman ${ }^{27}$, and quarter-order-differentiator (dash-dot-dash) as proposed by Brown and Stein ${ }^{5}$ and Chapman and $\mathrm{Smith}^{8}$. The frequency response was computed over the range of modulation frequencies used in the present study. The magnitudes were normalized to the largest value for each model. The transfer function expressions are given in Table II. Typical data points $(\boldsymbol{A})$ have been added to facilitate comparison between the models and experimental results. 


\section{TABLE II}

TRANSFER FUNCTIONS OF MECHANORECEPTORS

The gain factor, $A$, is constant with frequency and takes on different values in each case; $S$ is the Laplace operator.

\begin{tabular}{|c|c|}
\hline $\begin{array}{l}\text { Crayfish stretch receptor } \\
\left.\text { (Brown and Stein }{ }^{5}\right)\end{array}$ & $A \cdot S^{0.25}$ \\
\hline $\begin{array}{l}\text { Monkey knee joint } \\
\text { (Viernstein }{ }^{32} \text { ) }\end{array}$ & $A \cdot \frac{(S+0.152)}{(S+0.42)}$ \\
\hline $\begin{array}{l}\text { Golgi tendon organ } \\
\quad\left(\text { Houk and Henneman }{ }^{14}\right)\end{array}$ & $A \cdot \frac{(S+0.95)(S+13.65)}{(S+1.8)(S+25.0)}$ \\
\hline $\begin{array}{l}\text { Secondary muscle spindle } \\
\text { (Poppele and Bowman }\end{array}$ & $A \cdot \frac{(S+0.44)(S+11.3)}{(S+0.816)}$ \\
\hline
\end{tabular}

distinct qualitative difference in that the magnitude portion of the Bode plot is essentially flat for frequencies greater than $0.1 \mathrm{~Hz}$ and the phase angle predicted declines with frequency beyond $0.1 \mathrm{~Hz}$.

Empirical models are not necessarily related to the underlying physiological parameters of the receptor*. Such models are valuable, however, since they represent a compact summary of the frequency response of the receptor for a particular frequency range and allow predictions, in some cases, of the response of the receptor to other types of input functions. Furthermore, models can be used to develop our intuition of how such elemental responses might be processed by higher centers.

The transfer function gain extracted from the frequency response is influenced by both joint bias angle and input amplitude excursion, and seems to be roughly related to the slope of the static angle sensitivity curve at the point where the dynamic data were taken. The gain also decreased with increasing input excursion, which suggests a saturation type of non-linearity. The non-linearities noted are not of the type or severity to prevent a frequency analysis approach such as we have employed. Since a unique frequency response curve was not found, the transfer function derived from the data cannot be used to predict the response of the system to arbitrary inputs as in the case of standard linear analysis. Careful correction of the linear results to account for the effect of signal strength on system gain should produce approximately correct modelled responses however.

The tendency for some receptors to phase lock at high frequencies is also of interest. It would seem that phase-locking might effectively disable the receptor and prevent transfer of information above a certain input frequency. On the other hand the decoding process might switch to some other mode that makes use of these phaselocked responses. At this point we still do not know what parameters of the receptor signal are used (interpreted) by the system at higher levels.

The role of the slowly adapting joint receptor in joint angle perception is open

\footnotetext{
* Note added in proof. Recently, Thorson and Biederman-Thorson have related fractional order models to physiological parameters: Distributed relaxation processes in sensory adaptation, Science, 183 (1974) 161-172.
} 
to question. Several features of the receptor response (hysteresis, bell shaped sensitivity curves, phase-locking) appear to compromise their usefulness at lower levels of information processing. Careful behavioral and psychophysical experiments may determine joint angle perception capabilities in intact, unanesthetized animals.

The transfer function of the slowly adapting joint receptors is distinctive when compared to other types of joint receptors. Two types ${ }^{34,35}$ of rapidly adapting joint motion responses have been observed. A small number of responses exhibit Bode plot magnitude slopes of $20 \mathrm{~dB} /$ decade over a frequency range of $1-7 \mathrm{~Hz}$. A larger number of responses that exhibit a Bode plot magnitude slope of $40 \mathrm{~dB} /$ decade over a range of frequencies from 1 to $7 \mathrm{~Hz}$ have also been observed. This finding has led us to characterize these responses as velocity sensitive or acceleration sensitive, respectively. The frequency response characteristics of the various receptor types serve as useful templates against which we may compare responses obtained at higher levels. They allow one to characterize receptor type by observation of the slope of the Bode plot magnitude curve and also provide a mechanism for the evaluation of dynamic modifications imposed on the receptor information as it travels from level to level.

\section{APPENDIX}

The transfer function first used by Chapman and Smith ${ }^{8}$ for the cockroach mechanoreceptor and also by Brown and Stein ${ }^{5}$ for the crayfish stretch receptor has figured prominently in this work. The Bode plot predicted by their model is derived here.

The empirically fitted step response

$$
\mathrm{y}=\mathrm{ax} \mathrm{t}^{-\mathrm{k}}
$$

where $y$ is the receptor frequency, $x$ is the amplitude of the step, $t$ is time and a and $\mathrm{k}$ are constants, can be transformed to the $\mathrm{s}$ domain to get the transfer function

$$
\mathrm{G}(\mathrm{S})=\mathrm{a} \cdot \Gamma(1-\mathrm{k}) \mathrm{S}^{+\mathrm{k}}
$$

where $\mathrm{S}$ is the Laplace operator. The gamma function has a constant value determined by $(1-k)$. If $k$ were unity, the output would be the derivative of the input.

The frequency response may be obtained by replacing $S$ with $\mathrm{j} \omega$, where $\omega$ is the radian frequency and $j$ is the square root of minus one. We get:

$$
\mathrm{G}(\mathrm{j} \omega)=\mathrm{a} \cdot \Gamma(1-\mathrm{k})(\mathrm{j} \omega)^{\mathrm{k}}
$$

The usual Bode plot ordinate is in units of decibels: 20 times the logarithm of the magnitude of $G(j \omega)$. Since

$$
\begin{aligned}
|\mathrm{G}(\mathrm{j} \omega)| & =\mathrm{a} \cdot \Gamma(1-\mathrm{k}) \omega^{\mathrm{k}} \\
\mathrm{G}_{\mathrm{dB}} & =20 \log _{10}|\mathrm{G}(\mathrm{j} \omega)|=20 \log _{10}\left[\mathrm{a} \cdot \Gamma(1-\mathrm{k}) \omega^{\mathrm{k}}\right] \\
& =20 \log _{10}[\mathrm{a} \cdot \Gamma(1-\mathrm{k})]+20 \log _{10}\left(\omega^{\mathrm{k}}\right) \\
& =20 \log _{10}[\mathrm{a} \cdot \Gamma(1-\mathrm{k})]+20 \mathrm{k} \log _{10}(\omega)
\end{aligned}
$$


Note that the slope of the $\log -\log$ magnitude plot predicted by this model is:

$\mathrm{d} \mathrm{G}_{\mathrm{dB}} / \mathrm{d} \log _{10}(\omega)=20 \mathrm{k}=5 \mathrm{~dB} /$ decade if $\mathrm{k}=0.25$

The phase is also determined by the value of $\mathrm{k}$ :

$$
\begin{aligned}
\operatorname{Arg} \mathrm{G}(\mathrm{j} \omega) & =(\mathrm{j})^{\mathrm{k}}=\left(\mathrm{e}^{\mathrm{j} \pi / 2}\right)^{\mathrm{k}}=\mathrm{e}^{\mathrm{j} \pi / 2+\mathrm{n} 2 \pi, \mathrm{k}} \mathrm{n}=0, \pm 1, \pm 2, \ldots \\
& =\mathrm{e}^{\mathrm{j} \pi \mathrm{k} / 2+2 \pi \mathrm{nk},} \\
& =(\pi \mathrm{k} / 2+2 \pi \mathrm{nk}) \\
& =22.5 \text { for } \mathrm{k}=0.25, \mathrm{n}=0 \\
& =112.5^{\circ},-67.5^{\circ} \text { for } \mathrm{k}=0.25, \mathrm{n}=+1,-1
\end{aligned}
$$

Therefore, this analysis shows that the fractional order differentiator model, based on step response data, predicts a Bode plot with magnitude slope of $+5 \mathrm{~dB} /$ decade and constant phase lead of $22.5^{\circ}$, when $\mathrm{k}=0.25$ and $\mathrm{n}=0$.

It should be pointed out that this derivation is not mathematically rigorous in that the Laplace transform of $\mathrm{t}^{-\mathrm{k}}$ suffers from convergence difficulties. The Laplace transform can be generalized, in many cases, to have validity over a specified region, however.

Also, functions such as $\mathrm{S}^{0.25}$ have an infinite number of roots. In terms of the Bode plot this means that an infinite succession of phase profiles would satisfy the equation. For the case of the quarter-order-differentiator $22.5^{\circ}$ satisfies the equation. The principal value of $\mathrm{S}^{0.25}$ is the single valued function with the lowest root value, $22.5^{\circ}$ in this case. The principal value of $\mathbf{S}^{\mathbf{0 . 2 5}}$ is implied in our discussions.

The basic difficulty with such functions as the quarter-order-differentiator lies in its unrestricted use to predict system responses to arbitrary input functions. The range of applicability for transfer functions consisting of ratios of polynomials in ' $S$ ' is well established and one can use models of this type in the usual linear systems analysis and synthesis context, provided linearity or quasi-linearity criteria are met. However, generalized functions may predict system responses that are not meaningful or proper for a given input function.

The correct approach when generalized functions are used as empirical models is either to generalize carefully the Laplace transform to satisfy the necessary convergence conditions or to find a new empirical function that satisfies the requisite conditions. Within either of these two frameworks one can proceed to use the empirical model safely. The scope of this paper does not permit a detailed discussion of the problem; we merely seek to caution potential users of generalized functions in biological systems modelling. The empirical pairs $\mathrm{a}(\mathrm{t})^{-\mathrm{k}}$ and $\mathrm{a} \Gamma(1-\mathrm{k}) \mathrm{S}^{\mathrm{k}}$ seem to provide a good fit to a class of biological data and it is legitimate to use them for this purpose as long as the user is aware of the potential limitations in their applicability. The reader is referred to any advanced text on the Laplace transform for further discussion of this topic*.

\footnotetext{
* A more detailed treatment may be found in: Gel'fand, T. M., ANd Shilov, G. E., Generalized Functions, Vol. I, Properties and Operations, Academic Press, New York, 1964.
} 


\section{ACKNOWLEDGEMENTS}

The authors are grateful for the support of this work by U.S. Public Health Service Research Grant NS-08470 and a grant-in-aid of research from Sigma Xi.

W. D. McCall, Jr., was supported by U.S. Fublic Health Service Predoctoral Fellowship GM 42322, and is currently supported by Grant DE 02731-07. M. C. Farias was supported by Training Grant GM 01289.

\section{REFERENCES}

1 ANDrew, B. L., AND DodT, E., The deployment of sensory nerve endings at the knee joint of the cat, Acta physiol. scand., 28 (1954) 287-297.

2 Borsellino, A., Poppele, R. E., And Terzuolo, C. A., Transfer functions of the slowly adapting stretch receptor organ of crustacea, Cold Spr. Harb. Symp. quant. Biol., 30 (1965) 581-586.

3 BoYD, I. A., The histological structure of the receptors in the knee joint of the cat correlated with their physiological response, J. Physiol. (Lond.), 124 (1954) 476-488.

4 Boyd, I. A., AND Roberts, T. D. M., Proprioceptive discharges from the stretch receptors in the knee joint of the cat, J. Physiol. (Lond.), 122 (1953) 38-58.

5 Brown, M. C., AND Stein, R. B., Quantitative studies on the slowly adapting stretch receptor of the crayfish, Kybernetik, 3 (1966) 175-185.

6 Burgess, P. R., ANd Clark, F. J., Characteristics of knee joint receptors in the cat, J. Physiol. (Lond.), 203 (1969) 317-335.

7 Burgess, P. R., and Clark, F. J., Personal communication.

8 Chapman, K. M., and Smith, R. S., A linear transfer function underlying impulse frequency modulation in a cockroach mechanoreceptor, Nature (Lond.), 197 (1963) 699-701.

9 Clynes, M., Unidirectional rate sensitivity: A biocybernetic law of reflex and humoral systems as physiologic channels of control and communication, Ann. N.Y. Acad. Sci., 92 (1961) 946-969.

10 COHEN, L. A., Activity of knee joint proprioceptors recorded from the posterior articular nerve, Yale J. Biol. Med., 28 (1955) 225-232.

11 Eklund, G., AND Skoglund, S., On the specificity of the ruffini-like joint receptors, Acta physiol. scand., 49 (1960) 184-191.

12 Ellis, D. G., Temperature Effects on the Dynamic and Transient Behavior of Tendon, Ph. D. Thesis, Univ. of Michigan, 1970.

13 Gesink, J. W., AND Williams, W. J., A servo controlled linear actuator for bio-system studies, 21st Ann. Conf. Engr. Med. and Biol., (1968) Paper 18.5.

14 Houk, J. C., and Henneman, E., Response of Golgi tendon organs to active contractions of the soleus muscle of the cat, J. Neurophysiol., 30 (1967) 466-481.

15 Houk, J. C., AND Simon, W., Responses of Golgi tendon organs to forces applied to muscle tendon, J. Neurophysiol., 30 (1967) 1466-1481.

16 JANSEN, J. K. S., AND RudjoRd, T., On the silent period and Golgi tendon organs of the soleus muscle of the cat, Acta physiol. scand., 62 (1964) 364-379.

17 KAY, R. H., A reciprocal time interval display using transistor circuits, Electronic Engng., 37 (1965) 543-545.

18 KiRkwood, P. A., The frequency response of frog muscle spindles under various conditions, J. Physiol. (Lond.), 222 (1972) 135-160.

19 KNIGHT, B. W., Frequency response for sampling integrator and for voltage to frequency converter. In C. A. Terzuolo (Ed.), Conf. on Systems Analysis Approach to Neurophysiological Problems, Brainerd, Minn., 1969, pp. 61-72.

20 KNIGHT, B. W., Toyoda, J. I., AND DodGe, JR., F. A., A quantitative description of the dynamics of excitation and inhibition in the eye of Limulus, J. gen. Physiol., 56 (1970) 421-437.

21 Matthews, P. B. C., And Stein, R. B., The sensitivity of muscle spindle afferents to small sinusoidal changes of length, J. Physiol. (Lond.), 200 (1969) 723-743.

22 McCall, JR., W. D., AND Williams, W. J., Dynamic analysis of cat knee joint receptors, Fed. Proc., 30 (1971) 709. 
23 McKean, T. A., Poppele, R. E., Rosenthal, N. P., and Terzuolo, C. A., The biologically relevant parameter in nerve impulse trains, Kybernetik, 6 (1970) 168-170.

24 Mountcastle, V. (Ed.), Medical Physiology, C. V. Mosby, St. Louis, Mo., 1968.

25 Mountcastle, V., Poggto, G., and Werner, G., The relation of thalamic cell response to peripheral stimuli varied over an intensive continuum, $J$. Neurophysiol., 26 (1963) 807-834.

26 Poggio, G., and Mountcastle, V., The functional properties of ventrobasal thalamic neurons studies in unanesthetized monkeys, $J$. Neurophysiol., 26 (1963) 775-806.

27 Poppele, R. E., AND Bowman, R. J., Quantitative description of linear behavior of mammalian muscle spindles, J. Neurophysiol., 33 (1970) 59-72.

28 Roтн, P. R., Effective measurements using digital signal analysis, IEEE Spectrum, 8 (1971) 62-70.

29 RuDJORD, T. A., A second order mechanical model of muscle spindle primary endings, Kybernetik, 6 (1970) 205-213.

30 Skoglund, S., Anatomical and physiological studies of knee joint innervation in the cat, Acta physiol. scand., 36, Suppl. 124 (1956) 1-101.

31 Toyama, K., An analysis of impulse discharges from the spindle receptor, Jap. J. Physiol, 16 (1966) 113-125.

32 VieRnSTEIN, L. J., Dynamic properties of first order afferents responsive to joint rotation, 22nd Ann. Conf. Eng. Med. and Biol., (1967) Paper 4.7.

33 Williams, W. J., BeMent, S. L., Yin, T. C. T., and McCall, W. D., JR., Velocity and acceleration response in N. Gracilis to angular joint rotation, First Ann. Mtg., Soc. Neurosci., Wash., D.C., Oct. $28,1971$.

34 Williams, W. J., BeMent, S. L., Yin, T. C. T., and McCall, W. D., JR., Nucleus gracilis responses to knee joint motion: a frequency response study, Brain Research, 64 (1973) 123-140.

35 Williams, W. J., Gesink, J. W., and Stern, M. M., Biological system transfer-function extraction using swept-frequency techniques, Med. biol. Engng., 10 (1972) 609-620. 